November 13, 2017

\title{
PREDICTION OF COMPONENTS IN RANDOM SUMS
}

\author{
MUNEYA MATSUI
}

\begin{abstract}
We consider predictions of the random number and the magnitude of each iid component in a random sum based on its distributional structure, where only a total value of the sum is available and where iid random components are non-negative. The problem is motivated by prediction problems in a Poisson shot noise process. In the context, although conditional moments are best possible predictors under the mean square error, only a few special cases have been investigated because of numerical difficulties. We replace the prediction problem of the process with that of a random sum, which is more general, and establish effective numerical procedures. The methods are based on conditional technique together with the Panjer recursion and the Fourier transform. In view of numerical experiments, procedures work reasonably. An application in the compound mixed Poisson process is also suggested.
\end{abstract}

\section{Preliminaries}

Motivated by prediction problems in a Poisson shot noise process, we consider two types of problems for random sums of iid random variables (r.v. or r.v.'s for short). Let $N$ be a nonnegative integer-valued r.v. and denote an iid sequence of non-negative r.v's by $\left(X_{i}\right)_{i=1,2, \ldots}$ so that $S_{N}=\sum_{i=1}^{N} X_{i}$ denotes the total sum. The distributions of both $N$ and $X_{1}$ are assumed to be known. Our problem is how we could obtain the information of the number $N$ or each component $X_{i}$ when we only observe $S_{N}$. Although there are several methods for these quantities such as linear predictions $c S_{N}$ with $c$ some constant, our methods are those by conditional moments, which are minimizors of the mean square error. More precisely our focus is on the following two types of conditional moments:

$$
E\left[N^{k} \mid S_{N}\right] \quad \text { and } \quad E\left[X_{1}^{k} \mid S_{N}\right], \quad \text { for } k \in \mathbb{N},
$$

where $\left(X_{i}\right)$ may take both real and integer values and $\mathbb{N}$ denotes the set of natural numbers as usual.

This type of random sum $S_{N}$ has been studied for a long time and has applications in a variety of fields. One could find many examples in the book of Feller [5, XII] such as genetics, required service time, cosmic ray showers, and automobile accidents to name just a few. A large number of relevant researches have been conducted, including e.g. calculations for probability of $S_{N}$ (Sundt and Vernic [28]) or various limit theorems (see e.g. Gut [8] and consult a nice summary in Embrechts et al. [4, 2.5]). In recent years tail asymptotics have intensively studied, since accurate calculations of tail probabilities of $S_{N}$ are computationally quite expensive, while they are required in applications. See Jessen and Mikosch [9] for a survey with regularly varying tails and Goldie and Klüpperberg [6] for that with subexponential tails.

In this paper we do not go further into asymptotics but investigate precise calculations of quantities (1.1), which have not been studied yet except for some special cases (see Subsection 1.1). We rely on two numerical methods, i.e. the Panjer recursion and the Fourier method, which are useful tools for computing $P\left(S_{N}=n\right)$ and which are competitive ([3]). The Panjer recursion scheme

2010 Mathematics Subject Classification. Primary 60G50 ; Secondary 60G25, 60-08.

Key words and phrases. Random sum, prediction, conditional moments, Panjer recursion, Fourier transform, mixed Poisson process, Lévy processes.

Muneya Matsui's research is partly supported by the JSPS Grant-in-Aid for Young Scientists B (25870879). 
originated in Panjer [22] is known to be stable when $N$ belongs to the Panjer class in most cases ([23]). Meanwhile, the Fourier method could be applicable to general $N$, though it requires accurate numerical integrals. Here we show that these methods could be useful tools for computing quantities in (1.1) and establish efficient numerical procedures. Our methods do not depend on specific distributions on $N$ and $X_{1}$ and therefore could be applicable under general settings.

In the remainder of this section, we present a motivating application and its literature in Subsection 1.1 and introduce notations used in Subsection 1.2. In Section 2 random sums of discrete r.v.'s are treated, where computations of $E\left[N^{k} \mid S_{N}\right], k \in \mathbb{N}$ are investigated in Subsection 2.1 and those of $E\left[X_{1}^{k} \mid S_{N}\right]$ are studied in Subsection 2.2. Both the recursion method and the Fourier method are investigated. In Section 3, we consider random sums of non-negative continuous r.v.'s, where we take the Fourier approach for computations of both $E\left[N^{k} \mid S_{N} \leq x\right]$ and $E\left[X_{1}^{k} \mid S_{N} \leq x\right], x>0$. Finally in Section 4, numerical examples are given, which show that proposed methods work reasonably. As applications, we consider predictors for both the Poisson shot noise process and the compound mixed Poisson process.

1.1. Motivating application and its literature. A motivating example is prediction in the Poisson shot noise process of the form

$$
M(t)=\sum_{i=1}^{N(t)} L_{i}\left(t-T_{i}\right), \quad t>0,
$$

where $0<T_{1}<T_{2}<\cdots$ are points of a homogeneous Poisson $N(t)$ with intensity $\lambda>0$ and $\left(L_{j}\right)$ is a sequence of iid Lévy processes independent of $\left(T_{i}\right)$ and such that $L_{i}(t)=0$ a.s.t $\leq 0$. The process of this type has many applications in rather different areas (see [2], [29] [21] and [15]). One of important research topics is the prediction of future increments $M(t, t+s]:=M(t+s)-M(t), s, t>0$ based on the present observation $M(t)$. For example, in non-life insurance $M(t, t+s]$ is interpreted as the number or amount of future payment in the interval $(t, t+s]$ from an insurance company to the insured. Another interpretation is that $M(t, t+s]$ may describe the workload to be managed by a large computer network for sources in the interval $(t, t+s]$. Due to the properties of both Lévy and Poisson processes, the prediction of future increments $M(t, t+s]$ given $M(t)$ reduces to

$$
E[M(t, t+s] \mid M(t)]=E[N(s)] E\left[L_{1}(t+s-U)\right]+E\left[L_{1}(s)\right] E[N(t) \mid M(t)],
$$

where $U$ is a uniform r.v. on $(0, t)$ denoted by $U(0, t)$ independent of $\left(L_{i}\right)$. The proof of (1.3) is given in Appendix $\mathrm{A}$ or [16, (2.1)]. Here computations of $E[N(s)], E\left[L_{1}(s)\right]$ and $E\left[L_{1}(t+s-U)\right]$ are trivial. Since points $\left(T_{i}\right)$ of Poisson have the order statistic property, we can regard the sequence $\left(T_{i}\right)$ in the quantity $E[N(t) \mid M(t)]$ as that of iid $U(0, t)$ r.v.'s. Accordingly, taking $X_{i}:=L_{i}\left(t-T_{i}\right)$ and $N:=N(t)$ of $M(t)$, we obtain the form (1.1). Similarly, higher conditional moments $E\left[M(t, t+s]^{k}\right.$ $M(t)], k \in \mathbb{N}$ are obtained as functions of $E\left[N^{k}(t) \mid M(t)\right]$.

A series of papers [20], [10] and [16] assumes particular marginal distributions for $\left(L_{i}\right)$ such as Poisson or negative binomial, and exploits their specific properties to obtain the conditional moments. Although asymptotic behaviors of $E\left[N \mid S_{N}=k\right], k \rightarrow \infty$ have been studied in [10] and [25, 26], only limited distributions are treated. Our methods presented here require no particular assumptions on distributions of $N$ and $X_{1}$ and therefore could be applicable under more general settings than those of previous papers. 
1.2. Necessary notations and tools. Throughout we use the following notations related with generating functions. For a fixed r.v. $X$ and a non-negative function $f$ and $|u| \leq 1$,

$$
G_{X}(u):=E\left[u^{X}\right], \quad G_{f}(u):=\sum_{k=0}^{\infty} u^{k} f(k), \quad G_{d f}(u):=\int u^{x} d f(x),
$$

where the last one is defined as a Riemann-Stieltjes integral if exists. From these quantities we can obtain the Fourier (-Stieltjes) transforms $\phi_{\{\cdot\}}(u)=G_{\{\cdot\}}\left(e^{i u}\right)$. Note that we use generating functions not only for r.v.'s but also for discrete sequences (see [31]), though after a proper standardization, they are the same. We write $\mathbb{N}_{0}:=\{0,1,2, \ldots\}$ and $\mathbb{R}_{+}:=[0, \infty)$ in the sequel. Moreover, braces $\left\{\begin{array}{c}n \\ k\end{array}\right\}$ denote the Stirling numbers of the second kind (see [1, p.824]): the number of ways of partitioning a set of $n$ elements into $k$ non-empty sbsets.

We say that the probability mass function $q_{n}=P(N=n)$ belongs to the Panjer $(a, b)$ class if it satisfies

$$
q_{n}=\left(a+\frac{b}{n}\right) q_{n-1}, \quad n \in \mathbb{N} .
$$

for $a+b \geq 0$ and $a<1$ ([24, 20, p.122]). Poisson, negative binomial and binomial distributions belong to this class. For later use, we present the Panjer recursion formula (see [24, 20] for details and the proof).

Theorem 1.1. Suppose that $N$ belongs to the Panjer $(a, b)$ class and denote an iid sequence of non-negative integer-valued r.v.'s by $\left(X_{i}\right)$. Then

$$
\begin{array}{cc}
P\left(S_{N}=0\right)=E\left[P\left(X_{1}=0\right)^{N}\right], & n=0, \\
P\left(S_{N}=n\right)=\frac{1}{1-a P\left(X_{1}=0\right)} \sum_{j=1}^{n}\left(a+\frac{b j}{n}\right) P\left(X_{1}=j\right) P\left(S_{N}=n-j\right), & n \geq 1 .
\end{array}
$$

Here we let $0^{0}=1$ conventionally.

\section{RANDOM SUMS OF DISCRETE RANDOM VARIABLES}

2.1. Estimation of number of iid components. In this section calculations for conditional moments $E\left[N^{k} \mid S_{N}\right], k \in \mathbb{N}$ will be investigated, where iid random components $\left(X_{i}\right)$ are integervalued. In case $N$ belongs to the Panjer class, we apply the recursion formula to the calculation of $E\left[N^{k} \mid S_{N}\right]$. For general $N$, we consider the generating function of $E\left[N^{k} \mid S_{N}=\cdot\right]$ and then apply the inversion formula.

Throughout we denote the expectation of a r.v. $X$ over a measurable subset $A \subset \Omega$ by $E[X ; A]=$ $E\left[X I_{(X \in A)}\right]$ following Kallenberg [11, p.49]. Since we obtain $P\left(S_{N}\right)$ by Theorem 1.1, we mainly consider $E\left[N^{k} ; S_{N}\right], k \in \mathbb{N}$ which yields $E\left[N^{k} \mid S_{N}\right]=E\left[N^{k} ; S_{N}\right] / P\left(S_{N}\right)$.

Theorem 2.1. Let $N$ belong to the Panjer $(a, b)$ class and iid r.v.'s $\left(X_{i}\right)$ take values in $\mathbb{N}_{0}$. Let $C_{0}:=a P\left(X_{1}=0\right)$. Then, the restricted moments $m_{k}(\ell):=E\left[N^{k} ; S_{N}=\ell\right], k, \ell \in \mathbb{N}_{0}$ satisfy the recursion,

$$
\begin{aligned}
& m_{k}(0)=E\left[N^{k} P\left(X_{1}=0\right)^{N}\right], \\
& m_{k}(\ell)=\frac{1}{1-C_{0}}\left\{C_{0} \sum_{j=0}^{k-1}\left(\begin{array}{l}
k \\
j
\end{array}\right) m_{j}(\ell)+\sum_{j=1}^{\ell}\left(a+\frac{b j}{\ell}\right) P\left(X_{1}=j\right) \sum_{i=0}^{k}\left(\begin{array}{l}
k \\
i
\end{array}\right) m_{i}(\ell-j)\right\}, \quad \ell \geq 1,
\end{aligned}
$$

where $m_{0}(\ell)=P\left(S_{N}=\ell\right)$. 
Since for the calculation of $m_{k}(\ell)$, a combination of $m_{i}(\ell), i \leq k-1$ and $m_{i}(j), i \leq k, j \leq \ell-1$ is sufficient, we can recursively calculate the quantity.

Proof. The iidness of $\left(X_{i}\right)$ and independence between $N$ and $\left(X_{i}\right)$ yield

$$
m_{k}(0)=E\left[N^{k} ; S_{N}=0\right]=E\left[N^{k} E\left[I_{\left(S_{N}=0\right)} \mid N\right]\right]=E\left[N^{k} P\left(X_{1}=0\right)^{N}\right] .
$$

Next we consider $m_{k}(\ell), \ell \geq 1$. Conditioning argument and the Panjer $(a, b)$ class assumption yield

$$
m_{k}(\ell)=\sum_{i=1}^{\infty} i^{k} P\left(S_{i}=\ell\right) q_{i}=\sum_{i=1}^{\infty} P\left(S_{i}=\ell\right) i^{k}\left(a+\frac{b}{i}\right) q_{i-1}
$$

where $q_{n}=P(N=n), n \in \mathbb{N}_{0}$. Since $\left(X_{j}\right)$ are iid r.v.'s

$$
\left(a+\frac{b}{i}\right)=a+b \frac{1}{i} \sum_{j=1}^{i} E\left[\frac{X_{j}}{S_{i}} \mid S_{i}=\ell\right]=a+b E\left[\frac{X_{1}}{S_{i}} \mid S_{i}=\ell\right]=E\left[a+b \frac{X_{1}}{\ell} \mid S_{i}=\ell\right] .
$$

Moreover,

$$
E\left[a+\frac{b X_{1}}{\ell} \mid S_{i}=\ell\right]=\sum_{j=0}^{\ell}\left(a+\frac{b j}{\ell}\right) P\left(X_{1}=j \mid S_{i}=\ell\right)=\sum_{j=0}^{\ell}\left(a+\frac{b j}{\ell}\right) \frac{P\left(X_{1}=j\right) P\left(S_{i-1}=\ell-j\right)}{P\left(S_{i}=\ell\right)} .
$$

Substitution of this into $(a+b / i)$ of (2.2) and multiple interchanges of the order of summations give

$$
\begin{aligned}
m_{k}(\ell) & =\sum_{i=1}^{\infty} \sum_{j=0}^{\ell}\left(a+\frac{b j}{\ell}\right) P\left(X_{1}=j\right) P\left(S_{i-1}=\ell-j\right) i^{k} q_{i-1} \\
& =\sum_{j=0}^{\ell}\left(a+\frac{b j}{\ell}\right) P\left(X_{1}=j\right)\left\{\sum_{i=1}^{\infty} P\left(S_{i-1}=\ell-j\right) i^{k} q_{i-1}\right\} \\
& =\sum_{j=0}^{\ell}\left(a+\frac{b j}{\ell}\right) P\left(X_{1}=j\right) \sum_{i=0}^{\infty} P\left(S_{i}=\ell-j\right) \sum_{h=0}^{k}\left(\begin{array}{l}
k \\
h
\end{array}\right) i^{h} q_{i} \\
& =\sum_{j=0}^{\ell}\left(a+\frac{b j}{\ell}\right) P\left(X_{1}=j\right) \sum_{h=0}^{k}\left(\begin{array}{l}
k \\
h
\end{array}\right) m_{h}(\ell-j) \\
& =a P\left(X_{1}=0\right) m_{k}(\ell)+a P\left(X_{1}=0\right) \sum_{h=0}^{k-1}\left(\begin{array}{l}
k \\
h
\end{array}\right) m_{h}(\ell)+\sum_{j=1}^{\ell}\left(a+\frac{b j}{\ell}\right) P\left(X_{1}=j\right) \sum_{h=0}^{k}\left(\begin{array}{l}
k \\
h
\end{array}\right) m_{h}(\ell-j) .
\end{aligned}
$$

Thus we obtain the desired result.

If we take $k=1$ with $\ell \geq 1$ in Theorem 2.1, a rather simple expression is obtained

$$
m_{1}(\ell)=\frac{1}{1-C_{0}}\left\{C_{0} P\left(S_{N}=0\right)+\sum_{j=1}^{\ell}\left(a+\frac{b j}{\ell}\right) P\left(X_{1}=j\right)\left(P\left(S_{N}=\ell-j\right)+m_{1}(\ell-j)\right)\right\},
$$

which together with Theorem 1.1, yields the conditional expectation.

Next we consider the generating function for $m_{k}(\ell)$ with $N$ a general r.v. 
Proposition 2.2. Let $N$ be a r.v. on $\mathbb{N}_{0}$ and let $\left(X_{i}\right)$ be an iid sequence of r.v.'s on $\mathbb{N}_{0}$. Assume $E N^{k}<\infty, k \in \mathbb{N}$. Then the generating function of the truncated $k$-th moment $m_{k}(\ell)=E\left[N^{k} ; S_{N}=\right.$ $\ell]$ has the form

$$
G_{m_{k}}(u)=\sum_{j=1}^{k}\left\{\begin{array}{c}
k \\
j
\end{array}\right\} G_{X_{1}}^{j}(u) G_{N}^{(j)}\left(G_{X_{1}}(u)\right), \quad|u| \leq 1,
$$

where the quantities by braces \{\} denote the Striling number of the second kind, and $G_{Y}^{(j)}(u), j \in \mathbb{N}$ denotes the $j$-th derivative of $G_{Y}(x)$ at $x=u . G_{Y}^{(j)}(u), j \in \mathbb{N}$ denotes the $j$-th derivative of $G_{Y}(x)$ at $x=u$.

Proof. A direct calculation yields

$$
G_{m_{k}}(u)=\sum_{\ell=0}^{\infty} u^{\ell} E\left[N^{k} ; S_{N}=\ell\right]=E N^{k} \sum_{\ell=0}^{\infty} u^{\ell} P\left(S_{N}=\ell \mid N\right)=E N^{k} G_{X_{1}}^{N}(u),
$$

where $E N^{k}<\infty$ assures Fubini's theorem since $\left|G_{X_{1}}(u)\right| \leq 1$. We use the relation of the falling factorial $(x)_{k}=x(x-1) \cdots(x-k+1)$ and $x^{k}$,

$$
\sum_{j=1}^{k}\left\{\begin{array}{l}
k \\
j
\end{array}\right\}(x)_{j}=x^{k}, \quad\left\{\begin{array}{l}
k \\
0
\end{array}\right\}=0, \quad k>0,
$$

namely,

$$
E\left[N^{k} G_{X_{1}}^{N}(u)\right]=\sum_{j=1}^{k}\left\{\begin{array}{c}
k \\
j
\end{array}\right\} E\left[(N)_{j} G_{X_{1}}^{N}(u)\right]=\sum_{j=1}^{k}\left\{\begin{array}{c}
k \\
j
\end{array}\right\} G_{X_{1}}^{j}(u) E\left[(N)_{j} G_{X_{1}}^{N-j}(u)\right]=(2.3),
$$

where we change the order of derivatives and the summation, which is valid from $E N^{k}<\infty$ and $\left|G_{X_{1}}(u)\right| \leq 1$.

In order to obtain $m_{k}(\ell)=E\left[N^{k} ; S_{N}=\ell\right]$ from $G_{m_{k}}$, two methods are considered. One requires numerical integrations and the other needs derivatives of $G_{m_{k}}$ at the origin. Since $\left|G_{m_{k}}\left(e^{i v}\right)\right|^{2} \leq$ $\left(E N^{k}\right)^{2}<\infty$, we have $G_{m_{k}}\left(e^{i v}\right) \in L^{2}(-\pi, \pi)$. Then the Fourier expansion of $G_{m_{k}}\left(e^{i v}\right)$ is guaranteed and their coefficients satisfy formula

$$
m_{k}(\ell)=\frac{1}{2 \pi} \int_{-\pi}^{\pi} e^{-i \ell u} G_{m_{k}}\left(e^{i u}\right) \mathrm{d} u, \quad \ell \in \mathbb{N}_{0},
$$

which correspond to the inversion of the Fourier transform $G_{m_{k}}\left(e^{i v}\right)$. On the other hand, if we take derivatives of $G_{m_{k}}$ at the origin, we obtain $m_{k}(\ell)=\frac{1}{\ell !} G_{m_{k}}^{(\ell)}(0)$. In view of (2.3), however, the calculation of $G_{m_{k}}^{(\ell)}$ would yield additional complexities, though we may possibly find some efficient recursion methods for a limited class of $N$. The choice of the two methods depends on distributional assumptions on $N$ and $X_{1}$ and we need numerical experiments to judge which is better.

2.2. Estimation of magnitude of each iid component. In this subsection we consider the expected magnitude of r.v. $X_{1}^{k}, k \in \mathbb{N}$ under the observation of the total number $S_{N}$. Since the conditional moments minimize mean squared errors, we will consider $\chi_{k}=E\left[X_{1}^{k} \mid S_{N}\right], k \in \mathbb{N}$. Since the direct application of the Panjer recursion seems difficult for $\chi_{k}$ and easy for $\chi_{k+}:=E\left[X_{1}^{k} \mid S_{N+1}\right]$, we derive the recursion only for $\chi_{k+}$. Meanwhile, the Fourier approach is applied to both. 
Theorem 2.3. Let $N$ be a Panjer $(a, b)$ class distribution and let $\left(X_{i}\right)$ be a sequence of iid r.v.'s on $\mathbb{N}_{0}$. Assume $E X_{1}^{k}<\infty, k \in \mathbb{N}$, then the truncated $k$-th moment $\chi_{k+}(\ell)=E\left[X_{1}^{k} ; S_{N+1}=\ell\right], \ell \in \mathbb{N}$ has the form

$$
\begin{aligned}
& \chi_{k+}(1)=P\left(X_{1}=1\right) E\left[P\left(X_{1}=0\right)^{N}\right], \text { and for } \ell \geq 2, \\
& \chi_{k+}(\ell)=\ell^{k} P\left(X_{1}=\ell\right) P\left(S_{N}=0\right)+\frac{1}{1-a P\left(X_{1}=0\right)} \sum_{j=1}^{\ell-1} P\left(X_{1}=j\right)\left\{a \chi_{k+}(\ell-j)+\frac{b j^{k}}{\ell-j} \chi_{1+}(\ell-j)\right\} .
\end{aligned}
$$

Proof. For $\ell=1$, due to the iidness of $\left(X_{i}\right)$,

$$
\chi_{k+}(1)=E\left[X_{1}^{k} ; S_{N+1}=1\right]=P\left(X_{1}=1\right) E\left[P\left(X_{1}=0\right)^{N}\right] .
$$

Let $C_{1}:=1 /\left(1-a P\left(X_{1}=0\right)\right)$. For $\ell \geq 2$, the property of $N$ yields

$$
\begin{aligned}
\chi_{k+}(\ell) & =E\left[X_{1}^{k} ; S_{N+1}=\ell\right] \\
= & \sum_{j=1}^{\ell} j^{k} P\left(X_{1}=j\right) P\left(S_{N}=\ell-j\right) \\
= & \ell^{k} P\left(X_{1}=\ell\right) P\left(S_{N}=0\right)+\sum_{j=1}^{\ell-1} j^{k} P\left(X_{1}=j\right) C_{1} \sum_{m=1}^{\ell-j}\left(a+\frac{b m}{\ell-j}\right) P\left(X_{1}=m\right) P\left(S_{N}=\ell-j-m\right) \\
= & +\ell^{k} P\left(X_{1}=\ell\right) P\left(S_{N}=0\right)+C_{1}\left\{a \sum_{j=1}^{\ell-1} \sum_{m=1}^{\ell-j} j^{k} P\left(X_{1}=j\right) P\left(X_{1}=m\right) P\left(S_{N}=\ell-j-m\right)\right. \\
= & \quad \ell^{k} P\left(X_{1}=\ell\right) P\left(S_{N}=0\right)+C_{1}\left\{a \sum_{m=1}^{\ell-1} P\left(X_{1}=m\right) E\left[X_{1}^{k} ; S_{N+1}=\ell-m\right]\right. \\
& \left.+b \sum_{j=1}^{\ell-1} \frac{j^{k}}{\ell-j} P\left(X_{1}=j\right) E\left[X_{1} ; S_{N+1}=\ell-j\right]\right\},
\end{aligned}
$$

where in the third step, we use the Panjer recursion for $P\left(S_{N}=\ell-j\right)$. Finally, we arrange two sums and obtain the result.

For the calculation of $\chi_{k}=E\left[X_{1}^{k} \mid S_{N}\right]$ a direct application of the Panjer recursion seems difficult and alternatively we try the Fourier methods. For this we need the generating function of $\chi_{k}$.

Proposition 2.4. Let $N$ be a r.v. on $\mathbb{N}_{0}$ and let $\left(X_{i}\right)$ be an iid sequence of r.v.'s on $\mathbb{N}_{0}$. Assume $E X_{1}^{k}<\infty, k \in \mathbb{N}$, then the generating function of the truncated $k$-th moment $\chi_{k}(\cdot)=E\left[X_{1}^{k} ; S_{N}=\cdot\right]$ has the form

$$
G_{\chi_{k}}(u)=\sum_{j=1}^{k}\left\{\begin{array}{c}
k \\
j
\end{array}\right\} u^{j} G_{X_{1}}^{(j)}(u) \frac{G_{S_{N}}(u)}{G_{X_{1}}(u)}, \quad|u| \leq 1 .
$$

Proof. In view of

$$
\chi_{k}(\ell)=E\left[X_{1}^{k} ; S_{N}=\ell\right]=\sum_{j=1}^{\ell} j^{k} P\left(X_{1}=j\right) P\left(S_{N-1}=\ell-j\right),
$$


the function $\chi_{k}$ is the convolution of two non-negative functions $g_{1}(j):=j^{k} P\left(X_{1}=j\right)$ and $g_{2}(j):=$ $P\left(S_{N-1}=j\right)$. Since $G_{S_{N-1}}(u)=\sum_{j=1}^{\ell} u^{j} P\left(S_{N-1}=j\right)=E\left[G_{X_{1}}^{N-1}(u)\right]=G_{S_{N}}(u) / G_{X_{1}}(u)$, the generating function of $g_{1}(j)$ is enough. We use the relation of the falling factorial (2.4) and obtain

$$
E\left[X_{1}^{k} u^{X_{1}}\right]=\sum_{j=1}^{k}\left\{\begin{array}{c}
k \\
j
\end{array}\right\} E\left[\left(X_{1}\right)_{j} u^{X_{1}}\right]=\sum_{j=1}^{k}\left\{\begin{array}{c}
k \\
j
\end{array}\right\} u^{j} G_{X_{1}}^{(j)}(u)
$$

where we apply Fubini's theorem, which is possible by $E X_{1}^{k}<\infty$. Now the product of $G_{S_{N-1}}$ and $E\left[X_{1}^{k} u^{X_{1}}\right]$ yields the result.

Similarly as before, two methods are considered to obtain $\chi_{k}(\cdot)$ from $G_{\chi_{k}}$. One is to use derivatives at the origin, $\chi_{k}(\ell)=G_{\chi_{k}}^{(\ell)}(0) / \ell$ !,$\ell \geq 1$. The other is the inversion of generating function

$$
\chi_{k}(\ell)=\frac{1}{2 \pi} \int_{-\pi}^{\pi} e^{-i \ell u} G_{\chi_{k}}\left(e^{i u}\right) \mathrm{d} u, \quad \ell \in N_{0} .
$$

In view of (2.6), the former method requires some efficient algorithm for calculating derivatives of $G_{\chi_{k}}$, whereas for the second one, accurate numerical integrations are inevitable.

\section{RANDOM SUMS OF CONTINUOUS RANDOM VARIABLES}

In this section, we assume continuous distributions for an iid random sequence $\left(X_{i}\right)$ taking values on $\mathbb{R}_{+}$, while keeping $N$ to be r.v. on $\mathbb{N}_{0}$. Similarly as before we consider $E\left[N^{k} \mid S_{N}\right]$ and $E\left[X_{1}^{k} \mid\right.$ $\left.S_{N}\right], k \in \mathbb{N}$. Here the Fourier Stieltjes transform (FST for short) is our main tool.

3.1. Estimation of random number from random sum. We firstly consider $E\left[N^{k} \mid S_{N} \in\right.$ $[0, x]]=E\left[N^{k} \mid S_{N} \leq x\right]$ for $x \in \mathbb{R}_{+}$and $k \in \mathbb{N}$. We are starting to observe the integral equation as in [24, Sec. 4.4.3], which corresponds to the recursion formula when $X_{1}$ is a discrete distribution.

Theorem 3.1. Let $N$ be a Panjer $(a, b)$ class distribution and assume iid r.v.'s $\left(X_{i}\right)$ take values on $\mathbb{R}_{+}$with common distribution $F_{X_{1}}$. Then the restricted $k$-th moment to the Borel set by $\left\{S_{N} \leq x\right\}$, $m_{k}(x)=E\left[N^{k} ; S_{N} \leq x\right], k \in \mathbb{N}$ satisfies the integral equation,

$$
m_{k}(x)=a\left(m_{k} * F_{X_{1}}\right)(x)+\sum_{j=0}^{k-1}\left\{a\left(\begin{array}{c}
k \\
j
\end{array}\right)+b\left(\begin{array}{c}
k-1 \\
j
\end{array}\right)\right\}\left(m_{j} * F_{X_{1}}\right)(x), \quad x \geq 0,
$$

where the operation $*$ denotes the convolution as usual.

Proof. Since $N$ belongs to the Panjer $(a, b)$ class, we can write

$$
m_{k}(x)=\sum_{n=0}^{\infty} n^{k} q_{n} F_{X_{1}}^{*(n)}(x)=a \sum_{n=0}^{\infty}(n+1)^{k} q_{n} F_{X_{1}}^{*(n+1)}(x)+b \sum_{n=0}^{\infty}(n+1)^{k-1} q_{n} F_{X_{1}}^{*(n+1)}(x),
$$

where $F_{X_{1}}^{* n}(x)$ denotes the distribution of the $n$-th convolution of $X_{1}$. Using the binomial expansion and changing the order of summations, we obtain

$$
\begin{aligned}
m_{k}(x) & =a \sum_{n=0}^{\infty} \sum_{j=0}^{k}\left(\begin{array}{l}
k \\
j
\end{array}\right) n^{j} q_{n} F_{X_{1}}^{*(n+1)}(x)+b \sum_{n=0}^{\infty} \sum_{j=0}^{k-1}\left(\begin{array}{c}
k-1 \\
j
\end{array}\right) n^{j} q_{n} F_{X_{1}}^{*(n+1)}(x) \\
& =a \sum_{n=0}^{\infty} n^{k} q_{n} F_{X_{1}}^{*(n+1)}+\sum_{n=0}^{\infty}\left\{a \sum_{j=1}^{k-1}\left(\begin{array}{c}
k \\
j
\end{array}\right) n^{j} q_{n} F_{X_{1}}^{*(n+1)}(x)+b \sum_{j=0}^{k-1}\left(\begin{array}{c}
k-1 \\
j
\end{array}\right) n^{j} q_{n} F_{X_{1}}^{*(n+1)}(x)\right\}
\end{aligned}
$$




$$
=a\left(m_{k} * F_{X_{1}}\right)(x)+\sum_{j=0}^{k-1}\left\{a\left(\begin{array}{l}
k \\
j
\end{array}\right)+b\left(\begin{array}{c}
k-1 \\
j
\end{array}\right)\right\} \sum_{n=0}^{\infty} n^{j} q_{n} F_{X_{1}}^{*(n+1)}(x) .
$$

In view of expression (3.1), the integral equation seems useless to obtain $m_{k}(x)$ and we need additional techniques such as discretization of the density function of $X_{1}$ as in [24, Example (p.123)]. However, it is helpful to obtain the generating function of $m_{k}$ by providing an efficient recursion.

Lemma 3.2. Assume that $N$ belongs to the Panjer $(a, b)$ class and iid r.v's $\left(X_{i}\right)$ take values on $\mathbb{R}_{+}$ with common ch.f. $\phi_{X_{1}}$. Then the FST of $m_{k}(x)=E\left[N^{k} ; S_{N} \leq x\right], k \in \mathbb{N}$ has the following form

$$
\phi_{m_{k}}(u)=\frac{1}{1-a \phi_{X_{1}}(u)} \sum_{j=0}^{k-1}\left\{a\left(\begin{array}{l}
k \\
j
\end{array}\right)+b\left(\begin{array}{c}
k-1 \\
j
\end{array}\right)\right\} \phi_{m_{j}}(u) \phi_{X_{1}}(u), \quad u \in \mathbb{R} .
$$

The proof of Lemma is a straightforward calculation and we omit it. Notice that due to Lemma 3.2. $\phi_{m_{k}}(u)$ can be presented by a combination of $G_{N}\left(\phi_{X_{1}}(u)\right)$ and $\phi_{X_{1}}(u)$ since $\phi_{m_{0}}(u)=E\left[e^{i u S_{N}}\right]=$ $G_{N}\left(\phi_{X_{1}}(u)\right)$. For a general $N$, we directly calculate the FST of $m_{k}$.

Proposition 3.3. Let $N$ be a r.v. on $\mathbb{N}_{0}$ and let $\left(X_{j}\right)$ be an iid sequence of r.v.'s on $\mathbb{R}_{+}$. Assume $E N^{k}<\infty$ for $k \in \mathbb{N}$, then the FST of $m_{k}(x):=E\left[N^{k} ; S_{N} \leq x\right]$ has the form

$$
\int_{0}^{\infty} e^{i u x} \mathrm{~d} m_{k}(x)=E\left[N^{k} \phi_{X_{1}}^{N}(u)\right]=\sum_{j=1}^{k}\left\{\begin{array}{c}
k \\
j
\end{array}\right\} \phi_{X_{1}}^{j}(u) \phi_{N}^{(j)}\left(\phi_{X_{1}}(u)\right),
$$

where the left integral exists in the sense of the improper Riemann-Stieltjes integral.

Proof. Observe that $m_{k}(x)$ is a bounded non-decreasing function and $e^{i u x}$ is continuous for every $u \in \mathbb{R}$, then a Riemann-Stieltjes integral $\int_{0}^{M} e^{i u x} \mathrm{~d} m_{k}(x)$ exists for all $M>0$ [30, (2.24) Theorem]. Moreover, integration by parts (twice) and Fubini's theorem yield

$$
\begin{aligned}
\int_{0}^{M} e^{i u x} \mathrm{~d} m_{k}(x) & =\left[e^{i u x} m_{k}(x)\right]_{0}^{M}-\int_{0}^{M} i u e^{i u x} m_{k}(x) \mathrm{d} x \\
& =\left[e^{i u x} m_{k}(x)\right]_{0}^{M}-E\left[N^{k} \int_{0}^{M} i u e^{i u x} P\left(S_{N} \leq x \mid N\right) \mathrm{d} x\right] \\
& =\left[e^{i u x} m_{k}(x)\right]_{0}^{M}-E\left[N^{k}\left\{\left[e^{i u x} P\left(S_{N} \leq x \mid N\right)\right]_{0}^{M}-\int_{0}^{M} e^{i u x} \mathrm{~d} P\left(S_{N} \leq x \mid N\right)\right\}\right] \\
& =E\left[N^{k} \int_{0}^{M} e^{i u x} \mathrm{~d} P\left(S_{N} \leq x \mid N\right)\right],
\end{aligned}
$$

where in the third step, we use $m_{k}(x):=E\left[N^{k} P\left(S_{N} \leq x \mid N\right)\right]$ for all $x \geq 0$. To obtain the first equality of (3.2) take the limit $M \rightarrow \infty$ on both side, where in the right-hand side, the limit and expectation are exchangeable due to $E N^{k}<\infty$. Since the third equality follows similarly as in the proof of Proposition 2.2, we conclude the result.

The inversion of FST $\mathcal{F}^{-1}$ is well known [12, Theorem 4.4.1]: Let $\phi(u)$ be the FST of a bounded non-decreasing function $F(x)$, then $\mathcal{F}^{-1}$ is defined as

$$
F(x)=\mathcal{F}^{-1}[\phi(\cdot)](x)=\lim _{T \rightarrow \infty} \frac{1}{2 \pi} \int_{-T}^{T}\left[\left(e^{-i x t}-1\right) /-i t\right] \phi(t) \mathrm{d} t, \quad x>0 .
$$


Hence, $m_{k}(x)=\mathcal{F}^{-1}\left[\phi_{m_{k}}(\cdot)\right](x)$. When a r.v. $N$ is a Poisson with parameter $\lambda$, so that $a=0, b=\lambda$ in Theorem 3.1, we obtain $m_{1}(x)=\lambda\left(m_{0} * F_{X_{1}}\right)(x)$ and $m_{2}(x)=\lambda\left(m_{1} * F_{X_{1}}\right)(x)+m_{1}(x)$. Thus, it follows that

$$
m_{1}(x)=\lambda \mathcal{F}^{-1}\left[\phi_{X_{1}}(\cdot) e^{\lambda\left(\phi_{X_{1}}(\cdot)-1\right)}\right](x), \quad m_{2}(x)=\lambda^{2} \mathcal{F}^{-1}\left[\phi_{X_{1}}^{2}(\cdot) e^{\lambda\left(\phi_{X_{1}}(\cdot)-1\right)}\right](x)+m_{1}(x) .
$$

3.2. Estimation of magnitude of each iid component. A direct application of the Panjer recursion seems difficult for both $\chi_{k}$ and $\chi_{k+}$, and we alternatively invert FST of these functions. In order to obtain the FST, we represent $\chi_{k}$ and $\chi_{k+}$ in the form of a convolution.

Lemma 3.4. Let $N$ be a r.v. on $\mathbb{N}_{0}$ and $\left(X_{i}\right)$ is an iid sequence of r.v.'s on $\mathbb{R}_{+}$with common ch.f. $\phi_{X_{1}}$ such that $E X_{1}^{k}<\infty$. Then $\chi_{k}(x)=E\left[X_{1}^{k} ; S_{N} \leq x\right], k \in \mathbb{N}$ has the form,

$$
\chi_{k}(x)=\frac{1}{i^{k}} \mathcal{F}^{-1}\left[\phi_{S_{N}}(\cdot) \phi_{X_{1}}^{(k)}(\cdot) / \phi_{X_{1}}(\cdot)\right](x) .
$$

Proof. We exploit the expression

$$
\chi_{k}(x)=E\left[X_{1}^{k} ; S_{N} \leq x\right]=\int_{0}^{x} y^{k} P\left(S_{N-1} \leq x-y\right) \mathrm{d} P_{X_{1}}(y),
$$

which is the convolution of $P\left(S_{N-1} \leq \cdot\right)$ and $\int_{0} y^{k} \mathrm{~d} P_{X_{1}}(y)$. Since the ch.f. of $S_{N-1}$ is $\phi_{S_{N}}(u) / \phi_{X}(u)$, the conclusion is implied by the FST of $\int_{0}^{v} y^{k} \mathrm{~d} P_{X_{1}}(x)$ which is $\int_{0}^{\infty} e^{i u x} x^{k} \mathrm{~d} P_{X_{1}}(x)=i^{-k} \phi_{X_{1}}^{(k)}(u)$, where $E X_{1}^{k}<\infty$ assures the existence of $\phi_{X_{1}}^{(k)}(u)$.

The corresponding result for $E\left[X_{1}^{k} ; S_{N+1} \leq x\right]$ is obvious. Under the same condition of Lemma 3.4, we have $\chi_{k+}(x)=i^{-k} \mathcal{F}^{-1}\left[\phi_{S_{N}}(\cdot) \phi_{X_{1}}^{(k)}(\cdot)\right](x)$.

\section{NumERICAL EXAMPLES}

We prepare notations of distributions used in examples. Denote a Poisson distribution with parameter $\lambda$ by $\operatorname{Pois}(\lambda)$ and by $G e o(p)$, a geometric distribution with parameter $p$ of which probability is $P(X=k)=p q^{k}, q=1-p, k \in \mathbb{N}_{0}$. As usual write $X \sim \cdot$ if r.v. $X$ follows the distribution after the tilde. All computations are done with Mathematica ver. 9 of Wolfram.

Firstly a simple example of $E\left[N \mid S_{N}\right]$ is presented by setting $N \sim \operatorname{Pois}(\lambda)$ and $X_{1} \sim \operatorname{Pois}(\gamma)$. We examine two proposed methods for $m_{1}=E\left[N ; S_{N}\right]$, the recursion method and the Fourier inversion. For the probability of $S_{N}$, we use the ordinary recursion (Theorem 1.1), which yields

$$
P\left(S_{N}=\ell\right)= \begin{cases}E\left[P\left(X_{1}=0\right)^{N}\right]=e^{\lambda\left(e^{-\gamma}-1\right)}, & \ell=0, \\ \sum_{j=1}^{\ell} \frac{\lambda j}{\ell} P\left(X_{1}=j\right) P\left(S_{N}=\ell-j\right), & \ell \geq 1 .\end{cases}
$$

We apply Theorem 2.1 to obtain the recursion,

$$
m_{1}(\ell)= \begin{cases}E\left[N P\left(X_{1}=0\right)^{N}\right]=\lambda e^{-\gamma} e^{\lambda\left(e^{-\gamma}-1\right)}, & \ell=0, \\ \sum_{j=1}^{\ell} \frac{\lambda j}{\ell} P\left(X_{1}=j\right)\left\{P\left(S_{N}=\ell-j\right)+m_{1}(\ell-j)\right\}, & \ell \geq 1 .\end{cases}
$$

Another method for $m_{1}(\ell)$ is to apply (2.5) to the Fourier transform (Proposition 2.2), which is

$$
G_{m_{1}}\left(e^{i u}\right)=E N G_{X_{1}}^{N}\left(e^{i u}\right)=E N e^{\gamma\left(e^{i u}-1\right) N}=\lambda e^{\gamma\left(e^{i u}-1\right)} e^{\lambda\left(e^{\gamma\left(e^{i u}-1\right)}-1\right)} .
$$

In Figure 11, we plot $E\left[N \mid S_{N}=\ell\right]=m_{1}(\ell) / P\left(S_{N}=\ell\right), \ell \geq 0$ using both methods. Although they coincide when parameters are moderate, if either of parameters of Poisson for $N$ and $X_{1}$ is large, we observe instability for small $\ell$ in the Fourier approach (Figure 1 Right, squared dots), though for large $\ell$ there is no difference. 

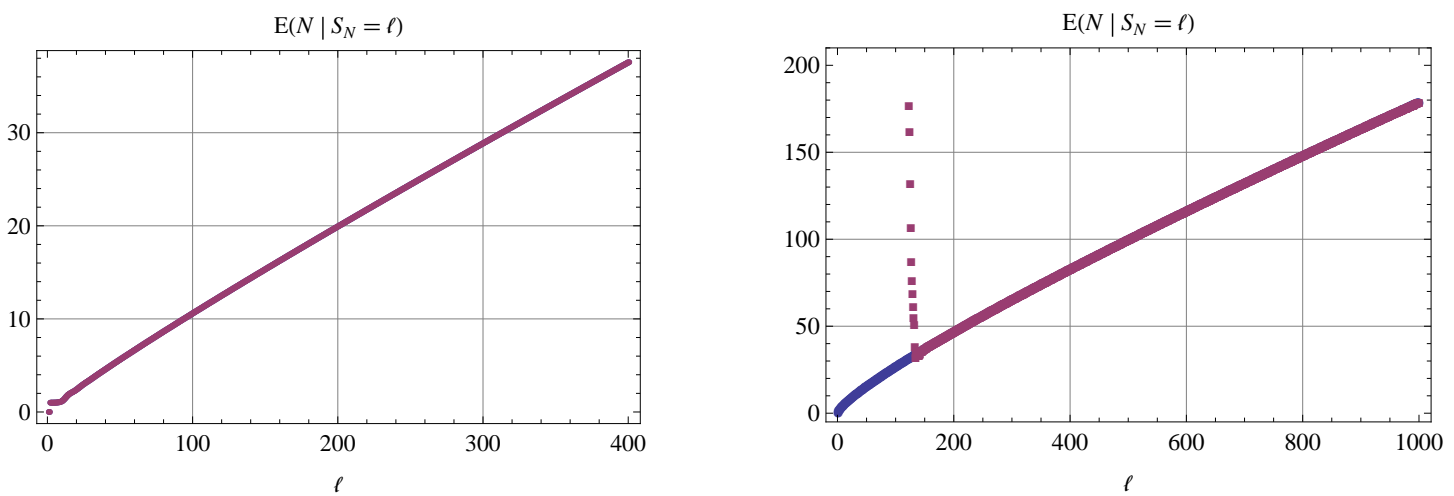

Figure 1. Left: the conditional moment $E\left[N \mid S_{N}=\ell\right], \ell \in[0,400]$ when Poisson parameters for $\left(N, X_{1}\right)$ are $(\lambda=20, \gamma=10)$. Right: $E\left[N \mid S_{N}=\ell\right], \ell \in[0,1000]$ under the setting $(\lambda=100, \gamma=5)$. Squared dots are values by the Fourier approach and round dots are those by the recursion method. In the former case values of both methods coincide. However, in the latter case instability is observed in the Fourier method for small $\ell$, though for large $\ell$ they coincide.

Next, we consider an example of $E\left[X_{1} \mid S_{N+1}\right]$ for the recursion and that of $E\left[X_{1} \mid S_{N}\right]$ with the Fourier transform, where $N \sim \operatorname{Pois}(\lambda)$ and $X_{1} \sim \operatorname{Geo}(p)$. The Panjer recursion is applied to both $P\left(S_{N}=\cdot\right)$ and $P\left(S_{N+1}=\cdot\right)$, for the latter of which we also use the convolution. For $\chi_{1+}(\ell)=E\left[X_{1} ; S_{N+1}=\ell\right]$, we use the recursion by Theorem 2.3 , i.e.

$$
\chi_{1+}(\ell)= \begin{cases}p q e^{-\lambda q}, & \ell=1, \\ \ell p q^{\ell} e^{-\lambda q}+\sum_{j=1}^{\ell-1} p q^{j} \frac{\lambda j}{\ell-j} \chi_{1+}(\ell-j), & \ell \geq 2 .\end{cases}
$$

For $\chi_{1}(\ell)=E\left[X_{1} ; S_{N}=\ell\right]$, the inversion of the Fourier transform (2.5) is applied to

$$
G_{m_{1}}\left(e^{i u}\right)=\frac{q e^{i u}}{1-q e^{i u}} \exp \left\{\lambda \frac{q\left(1-e^{i u}\right)}{q e^{i u}-1}\right\} .
$$

In Figure 2, we plot $E\left[X_{1} \mid S_{N}=\ell\right]$ and $E\left[X_{1} \mid S_{N+1}=\ell\right]$ for $\ell \geq 1$. Since the graphs show very similar curves for a moderate setting of parameters, we conclude that both methods work properly. However the instability is again observed in the Fourier approach (Figure 2: Right, squared dots) when the parameter $\lambda$ is large and $\ell$ is small.

4.1. Prediction in Poisson shot noise process. We pursue the prediction $E[M(t, t+s] \mid M(t)], t, s>$ 0 of the model (1.2), i.e. calculate the quantity $E[N(t) \mid M(t)]$ in (1.3). As mentioned, since the order of $\left(T_{j}\right)$ in (1.2) does not change the distributional relation of $N(t)$ and $M(t)$, by the order statistics property of the Poisson, we may consider the model $M(t):=\sum_{k=1}^{N(t)} L_{k}\left(t-U_{k}\right)$ with the iid $U(0, t)$ sequence $\left(U_{i}\right)$, and then study $E[N(t) \mid M(t)]$. We assume that the processes $L_{k}$ 's are iid compound Poisson processes such that the generic process $L$ has the form $L(t)=\sum_{j=1}^{N_{0}(t)} Y_{j}$, where $N_{0}(t) \sim \operatorname{Pois}(\gamma t)$, and $\left(Y_{j}\right)$ denotes an iid sequence of non-negative jump sizes.

Now by setting $N:=N(t)$ and $X_{i}:=L_{i}\left(t-U_{i}\right), i \in \mathbb{N}$, the calculation of $E[N(t) \mid M(t)]$ can be considered in the framework of $E\left[N \mid S_{N}\right]$. For the probability of $X_{1}$, since $N_{0}\left(t-U_{1}\right)$ does not belong to the Panjer class, we take the Fourier approach. For this we need ch.f. of $X_{1}:=L_{1}\left(t-U_{1}\right)$, which is

$$
E\left[e^{i u X_{1}}\right]=E\left[e^{i u L\left(t-U_{1}\right)}\right]=E\left[e^{i u \sum_{j=1}^{N_{0}\left(t-U_{1}\right)} Y_{j}}\right]=\frac{e^{\gamma(t-1)\left(\phi_{Y_{1}}(u)-1\right)}-e^{\gamma t\left(\phi_{Y_{1}}(u)-1\right)}}{\gamma\left(1-\phi_{Y_{1}}(u)\right)},
$$



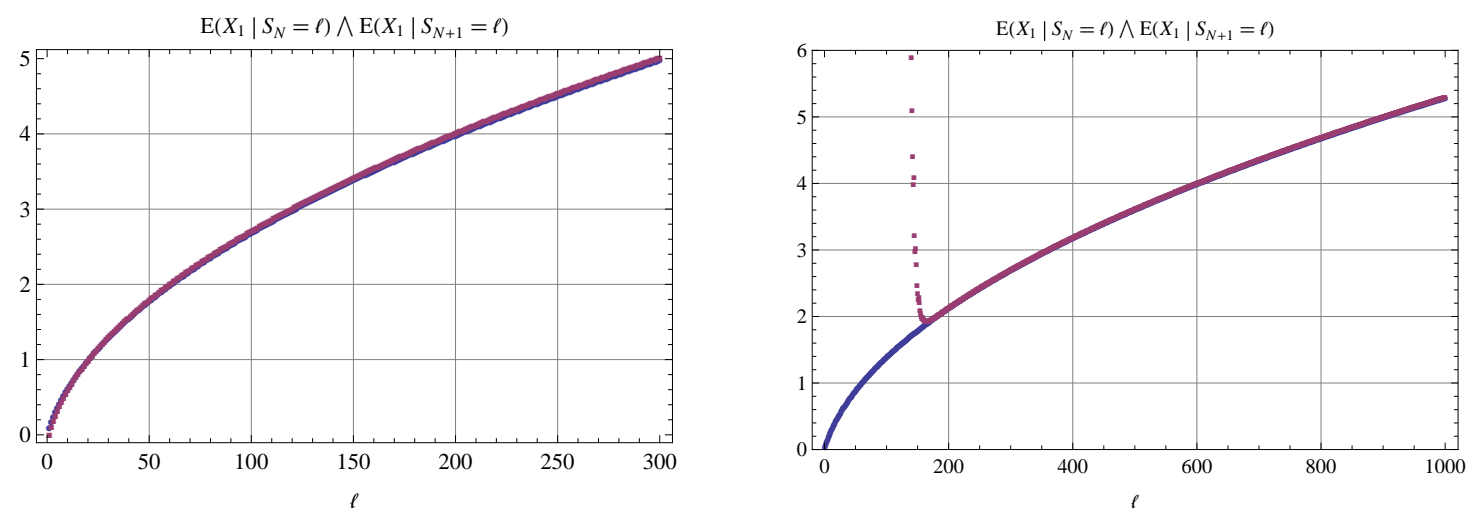

FIGURE 2. Left: the conditional moments $E\left[X_{1} \mid S_{N}=\ell\right]$ (square dots) and $E\left[X_{1} \mid\right.$ $\left.S_{N+1}=\ell\right]$ (round dots) for $\ell \in[1,300]$ when parameters of $\operatorname{Pois}(\lambda)$ and $G e o(p)$ for $\left(N, X_{1}\right)$ are $(\lambda=40, p=0.25)$ respectively. Right: the same quantities of the left but with $\ell \in[1,1000]$ and $(\lambda=150, p=0.2)$. In both graphs these quantities present quite similar curves for large $\ell$. However, in the right graph instability occurs in small $\ell$ of $E\left[X_{1} \mid S_{N}=\ell\right]$ (the Fourier approach).
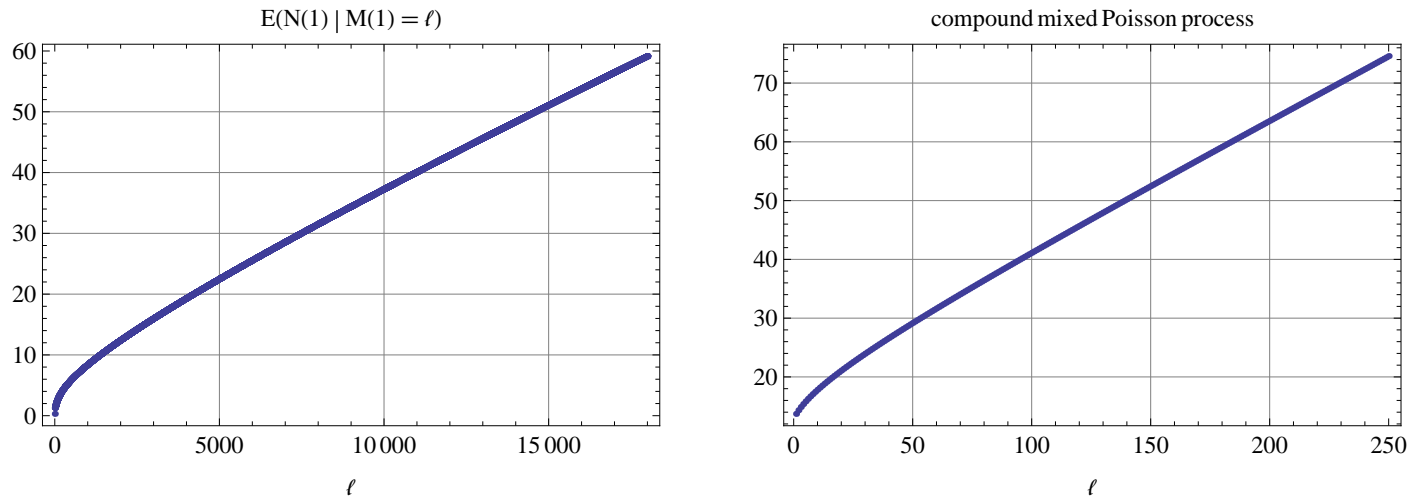

FIGURE 3. Left: we plots $E[N(1) \mid M(1)=\ell], \ell \in[0,18000]$ which is the quantity in the predictor of a Poisson shot noise process of (1.3) with $t=1$. Right: we plot the predictor of a compound mixed Poisson process, $E[Z(1,2] \mid Z(1)=\ell], \ell \in[0,250]$. In both graphs one see the non-linear curves which show that the linear estimations are insufficient.

where $\phi_{Y_{1}}(u)$ is the ch.f. of $Y_{1}$. Thus after putting $Y_{1} \sim \operatorname{Pois}(\mu)$ so that $\phi_{Y_{1}}(u)=e^{\mu\left(e^{i u}-1\right)}$ we obtain the probability of $X_{1}$ by the Fourier inversion. For simplicity, we set $t=1$, i.e. consider $E[N(1) \mid M(1)]$, and apply Theorem 2.1 or equivalently apply the recursions (4.1) and (4.2) with initial values $P\left(S_{N}=0\right)=e^{\lambda\left(P\left(X_{1}=0\right)-1\right)}$ and $m_{1}(0)=E\left[N P\left(X_{1}=0\right)^{N}\right]=\lambda P\left(X_{1}=0\right) P\left(S_{N}=0\right)$.

In Figure 3 (left), we plots $E[N(1) \mid M(1)=\ell]$ for $\ell \in[0,18000]$ with $\gamma=100, \mu=5$ and $\lambda=30$. In view of the graph, our computational method seems to work well, and one can see a non-linear curve which shows that the linear estimation of $N(t)$ by $M(t)$ is insufficient.

4.2. Prediction in compound mixed Poisson process. We consider an example of the compound mixed Poisson process mixed by a Gamma r.v. called compound Pólya process [7, Ex. 4.1]. Let $\bar{N}(t):=\pi(\theta \Lambda(t))$ denotes a mixed Poisson process where $\pi(t)$ be a homogeneous Poisson process 
with intensity 1 on $[0, \infty), \Lambda(t)$ is an intensity measure and $\theta$ is a Gamma $(\alpha, \beta)$ r.v. of which density is $f_{\theta}(x)=\frac{\beta^{\alpha}}{\Gamma(\alpha)} x^{\alpha-1} e^{-\beta x}$. Then the process has the form $Z(t)=\sum_{j=1}^{\bar{N}(t)} X_{j}, t>0$, where $X_{j}$ 's are iid r.v.'s on $\mathbb{N}_{0}$ or $\mathbb{R}_{+}$such that $\bar{N}$ and $\left(X_{j}\right)$ are independent. Since the $\sigma$-fields $\mathcal{G}_{t}$ by $\{\bar{N}(t), \bar{N}(t, t+s], Z(t)\}, t, s>0$ and $\mathcal{H}_{t}$ by $\{\bar{N}(t), Z(t)\}$ are finer than that by $\{Z(t)\}$, the conditional expectation of increments $Z(t, t+s]:=Z(t+s)-Z(t)$ given $Z(t)$ has

$$
\begin{aligned}
E[Z(t, t+s] \mid Z(t)] & =E\left[E\left[E\left[Z(t, t+s] \mid \mathcal{G}_{t}\right] \mid \mathcal{H}_{t}\right] \mid Z(t)\right] \\
& =E\left[X_{1}\right] E\left[E\left[\bar{N}(t, t+s] \mid \mathcal{H}_{t}\right] \mid Z(t)\right] \\
& =E\left[X_{1}\right] E[E[\bar{N}(t, t+s] \mid \bar{N}(t)] \mid Z(t)],
\end{aligned}
$$

where in the third step we use the conditional independence of $\bar{N}(t, t+s]$ and $Z(t)$ given $\bar{N}(t)([11$, Prop. 6.6]). Since

$$
\begin{aligned}
E[\bar{N}(t, t+s] \mid \bar{N}(t)=m] & =\sum_{k=0}^{\infty} k \frac{\Lambda^{k}(t, t+s]}{k !} \frac{E\left[\theta^{k+m} e^{-\theta \Lambda(t+s)}\right]}{E\left[\theta^{m} e^{-\theta \Lambda(t)}\right]} \\
& =\Lambda(t, t+s] \frac{E\left[\theta^{m+1} e^{-\theta \Lambda(t)}\right]}{E\left[\theta^{m} e^{-\theta \Lambda(t)}\right]} \\
& =\Lambda(t, t+s] \frac{\alpha+m}{\Lambda(t)+\beta},
\end{aligned}
$$

where in the second step we exchange the infinite sum and the expectation operator (see also [7, (1.4)]), we proceed the calculation (4.3) to get

$$
E[Z(t, t+s] \mid Z(t)]=\frac{E\left[X_{1}\right] \Lambda(t, t+s]}{\Lambda(t)+\beta}(\alpha+E[\bar{N}(t) \mid Z(t)]) .
$$

Now let $\Lambda(t):=t, \beta:=1, \alpha:=7$ and $X_{1} \sim$ Geo (1/4), we obtain

$$
E[Z(t, t+s] \mid Z(t)]=\frac{3 s}{1+t}(7+E[\bar{N}(t) \mid Z(t)]) .
$$

Since $\bar{N}(t)$ does not belong to the Panjer class, we apply the Fourier approach. Due to Proposition 2.2 together with

$$
G_{S_{N}}(u)=E\left[G_{X_{1}}^{\bar{N}(t)}(u)\right]=\frac{1}{1+t\left(1-G_{X_{1}}(u)\right)} \quad \text { and } \quad G_{m_{1}}(u)=E\left[\bar{N} G_{X_{1}}^{\bar{N}}(u)\right]=\frac{7 G_{X_{1}}(u)}{\left\{1+t\left(1-G_{X_{1}}(u)\right)\right\}^{8}},
$$

we obtain the quantity $E[\bar{N}(t) \mid Z(t)]$ by the inversion formula (2.5). In Figure 3 (right), we plot $E[Z(t, t+s] \mid Z(t)=\ell], \ell \in[0,250]$ with $s=t=1$, where one would again observe a non-linear curve.

\section{Appendix A. Calculation of 1.3}

For the calculation of (1.3), we use the following properties.

(a). By definition, the $\sigma$-field by $M(t)$ is included in the $\sigma$-filed by $\left(L_{k}\left(t-T_{k}\right)\right)$ and $\left(T_{k}\right)$.

(b). Since $N(t)=\sum_{k=1}^{\infty} I_{\left(T_{k} \leq t\right)}$, the $\sigma$-field by $N(t)$ is included in the $\sigma$-field by $\left(T_{k}\right)$.

(c). By the order statistics property of a Poisson, given $N(t)$ and $N(t+s)$, the set of points

$\left(T_{k}\right) \in(0, t]$ and the set of points $\left(T_{k}\right) \in(t, t+s]$ are independent.

(d). Given $N(t, t+s]$, points $\left(T_{k}\right) \in(t, t+s]$ are mutually independent.

(e). Stationary and independent increments of Lévy processes. 
By a multiple use of iterated property of the conditional expectation [11, Theorem 6.1 (vii)], detailed the calculation of (1.3) is

$$
\begin{aligned}
E & {[M(t, t+s] \mid M(t)] } \\
= & E\left[\sum_{j=N(t)+1}^{N(t+s)} L_{j}\left(t-T_{j}, t+s-T_{j}\right] \mid M(t)\right]+E\left[\sum_{j=1}^{N(t)} L_{j}\left(t-T_{j}, t+s-T_{j}\right] \mid M(t)\right] \\
= & E\left[\sum_{j=N(t)+1}^{N(t+s)} E\left[L_{j}\left(t-T_{j}, t+s-T_{j}\right] \mid N(t), N(t+s),\left\{\left(T_{k}\right),\left(L_{k}\left(t-T_{k}\right)\right)\right\}_{k: T_{k} \leq t}\right] \mid M(t)\right] \\
& +E\left[\sum_{j=1}^{N(t)} E\left[L_{j}\left(t-T_{j}, t+s-T_{j}\right] \mid\left\{\left(T_{k}\right),\left(L_{k}\left(t-T_{k}\right)\right)\right\}_{k: T_{k} \leq t}\right] \mid M(t)\right] \\
= & E\left[\sum_{j=N(t)+1}^{N(t+s)} E\left[L_{j}\left(t+s-T_{j}\right) I_{\left(t<T_{j} \leq t+s\right)} \mid N(t), N(t+s)\right] \mid M(t)\right] \\
& +E\left[\sum_{j=1}^{N(t)} E\left[L_{j}\left(t-T_{j}, t+s-T_{j}\right] \mid T_{j}, L_{j}\left(t-T_{j}\right)\right] \mid M(t)\right] \\
= & E\left[\sum_{j=N(t)+1}^{N(t+s)} E[L(t+s-U)] \mid M(t)\right]+E\left[\sum_{j=1}^{N(t)} E[L(s)] \mid M(t)\right] \\
= & E[N(t, t+s] \mid M(t)] E[L(t+s-U)]+E[L(s)] E[N(t) \mid M(t)],
\end{aligned}
$$

where in the second step, the properties (a) and (b) are used, and in the third step, we exploit (c) and (e) so that the conditional independence of $\left(L_{j}\left(t-T_{j}, t+s-T_{j}\right]\right)_{j: t<T_{j} \leq t+s}$ and $\left\{\left(T_{i}\right),\left(L_{i}\left(t-T_{i}\right)\right)\right\}_{i: T_{i} \leq t}$. In the fourth step we use (d) and (e). Finally since the quantity $N(t, t+s]$ is independent of the $\sigma$-field $\mathcal{F}_{t}$ constructed by all available set before $t$, the conclusion holds.

Acknowledgment: The author would like to thank Prof. Tomasz Rolski for fruitful discussion of the topic and hospitality when he visited the mathematical institute in the University of Wroclaw.

\section{REFERENCES}

[1] Abramowitz, M. and Stegun, I. A. (1972) Handbook of Mathematical Functions with Formulas, Graphs, and Mathematical Tables. National Bureau of Standards Applied Mathematics Series 55. Tenth Printing.

[2] Bartlett, M.S. (1963) The spectral analysis of point processes. J. R. Statist. Soc. Ser. B. Stat. Methodol. 25, 264-296.

[3] Embrechts, P. And Frei, M. (2009) Panjer recursion versus FFT for compound distributions. Math. Methods Oper. Res. 69, 497-508.

[4] Embrechts, P., Klüppelberg, C. and Mikosch, T. (1997) Modelling Extremal Events for Insurance and Finance. Springer, Berlin.

[5] Feller, W. (1968) An Introduction to Probability Theory and its Applications. Vol. I, 3rd ed. Wiley, New York.

[6] Goldie, C. And Kl uppelberg (1998) Subexponential distributions. In: Adler, R. Feldman, R. and Taqqu, M. S. (Eds.) A Practical Guide to Heavy Tails: Statistical Techniques and Applications, 435-460. Birkhäuser, Boston.

[7] Grandell, J. (1997) Mixed Poisson Processes. Chapman and Hall, London.

[8] Gut, A. (2009) Stopped Random Walks : Limit Theorems and Applications. 2rd ed. Springer, New York.

[9] Jessen, A.H., Мiкosch, T. (2006) Regulary varying functions. Publications de L'institut Mathématique 80, 171192. 
[10] Jessen, A.H., Mikosch, T. and Samorodnitsky, G. (2011) Prediction of outstanding payments in a Poisson cluster model. Scand. Actuar. J. 2011, 214-237.

[11] Kallenberg, O. (2002) Foundations of Modern Probability. 2nd ed. Springer, New York.

[12] Kawata, T. (1972) Fourier Analysis in Probability Theory. Academic Press, New York.

[13] Klüppelberg, C. and Mikosch, T. (1995) Explosive Poisson shot noise processes with applications to risk reserves. Bernoulli 1, 125-147.

[14] Klüppelberg, C. And Mikosch, T. (1995) Delay in claim settlement and ruin probability approximations. Scand. Actuar. J. 1995, 154-168.

[15] Lewis, P.A. (1964) A branching Poisson process model for the analysis of computer failure patterns. J. R. Stat. Soc. Ser. B Stat. Methodol. 26, 398-456.

[16] Matsui, M. and Mikosch, T. (2010) Prediction in a Poisson cluster model. J. Appl. Probab. 47, $350-366$.

[17] Matsui, M. (2011) Prediction in a Poisson cluster model with multiple cluster processes. Scand. Actuar. J. 2015, $1-31$.

[18] Matsui, M. (2014) Prediction in a non-homogeneous Poisson cluster model. Insurance Math. Econom., 55, 1017.

[19] Matsui, M. and Rolski, T. (2014) Prediction in a mixed Poisson cluster model, preprint.

[20] Мiкоsch, T. (2009) Non-Life Insurance Mathematics. An Introduction with the Poisson Process. 2nd ed. Springer, Heidelberg.

[21] Neyman, J. and Scott, E.L. (1958) A statistical approach to problems of cosmology. J. R. Stat. Soc. Ser. B. Stat. Methodol. 20, 1-43.

[22] Panjer, H.H. (1981) Recursive evaluation of a family of compound distributions. Astin Bull. 12, $22-26$.

[23] Panjer, H.H. and Wang, S. (1993) On the Stability of Recursive Formulas. Astin Bull. 23, $227-258$.

[24] Rolski, T., Schmidli, H., Schmidt, V. And Teugels, J. (1999) Stochastic Processes for Insurance and Finance, Wiley, New York.

[25] Rolski, T. and TomaneK, A. (2011) Asymptotics of conditional moments of the summand in Poisson compounds. J. Appl. Probab. 48A, 65-76.

[26] Rolski, T. and Tomanek, A. (2014) A continuous-time model for claims reserving. Applicationes Mathematicae 41, 277-300.

[27] Sato, K.-I. (1999) Lévy Processes and Infinitely Divisible Distributions. Cambridge University Press, Cambridge, UK.

[28] Sundt, B. And Vernic, R. (2009) Recursions for convolutions and compound distributions with insurance applications. Springer, Berlin.

[29] Vere-Jones, D. (1970) Stochastic models for earthquake occurrences. J. R. Stat. Soc. Ser. B. Stat. Methodol., 32, 1-62.

[30] Wheeden R.L. and Zygmund A. (1977) Measure and integral: an introduction to real analysis. CRC Press, Bca Raton.

[31] WiLf H.S. (1993) Generatingfunctionology. 2nd. ed. Academic Press, San Diego.

Department of Business Administration, Nanzan University, 18 Yamazato-cho Showa-ku Nagoya, 466-8673, Japan E-mail address: mmuneya@nanzan-u . ac . jp 